Modelling of bond between three-wire strands and concrete

R. Bolmsvik and K. Lundgren

Published in Magazine of Concrete Research, see journal homepage http://www.icevirtuallibrary.com/content/journals

"Permission is granted by ICE Publishing to print one copy for personal use. Any other use of these PDF files is subject to reprint fees." 


\title{
Modelling of bond between three-wire strands and concrete
}

\author{
R. Bolmsvik* and K. Lundgren $\dagger$ \\ Abetong Teknik AB, Chalmers University of Technology
}

The bond between strands and concrete is of importance for prestressed concrete. The research presented in the current paper aims at a better understanding of the bond mechanism, and of how different detailings of the strand interface affect the behaviour. A bond model for three-wire strands was established and calibrated by use of pullthrough tests. The results from finite element (FE) analyses with the bond model and the tests were used in parallel. It was found that adhesion, friction and the ability to develop normal stresses determine the bond response of the strand; consequently, they were used as input parameters in the bond model. How different detailing of the strand surface affects these parameters, and the influence on the bond mechanism, are shown. For example, adhesion has the strongest influence on the initial bond response in the cases of smooth and indented strands. Regarding indented strands, the maximum bond capacity is determined by the strand indentation. The knowledge gained can be used to design the strands for a certain bond behaviour.

\section{Introduction}

The bond between strands and concrete is crucial for the behaviour and capacity of precast, prestressed concrete members. Through the years, the design of different precast, prestressed concrete members has been developed and adjusted in order to obtain a high load capacity in combination with a slim geometry. It has been found that the bond behaviour of strands can be strongly affected, both negatively and positively, by variations in properties of the concrete or the strand surface. This implies that $(a)$ a sound knowledge of the present bond mechanisms and $(b)$ detailed analyses of each concrete member design are needed in order to ensure the behaviour and the load capacity. Non-linear finite element (FE) analysis, where a model for the behaviour of the strand-concrete interface is used, is a powerful tool for this type of study.

This paper presents a bond model that has been developed for prestressing strands. The model is based on a theoretical model developed to describe the bond mechanism between deformed bars and concrete; see

\footnotetext{
* Abetong Teknik AB, SE-351 03, Växjö, Sweden.

$\dagger$ Department of Structural Engineering and Mechanics, Chalmers University of Technology, SE-412 96, Göteborg, Sweden.
}

(MCR 41332) Paper received 15 October 2004; accepted 16 November 2005 .
Lundgren and Gylltoft ${ }^{1}$ or Lundgren. ${ }^{2}$ Pull-through and push-in tests of three-wire strands with an indented or a smooth wire surface were conducted in order to investigate the present bond mechanism for strands, see Gustavson. ${ }^{3,4,5}$ The test results were also used as reference data during the calibration of the established bond model. Hence, the model can be used to model an explicit bond behaviour of a certain strand configuration through calibration with reference data. However, the primary use is to identify the mechanisms behind the bond behaviour and then use the acquired knowledge to design the strands in such a way that the desired bond behaviour is obtained. The FE program DIANA was used for all analyses.

\section{Bond model}

In the FE program DIANA, interface elements are available that describe a relation between the traction $\mathbf{t}$ and the relative displacement $\mathbf{u}$ in the interface. These interface elements are used at the surface between the strands and the concrete to describe the bond mechanism. Both the strands and the concrete are modelled with solid elements. The physical interpretations of the variables $t_{\mathrm{n}}, t_{\mathrm{t}}, u_{\mathrm{n}}$ and $u_{\mathrm{t}}$ are shown in Figure 1. The interface elements have, initially, a thickness of zero.

The theoretical model used to describe the bond mechanism is mainly the same as that developed and 


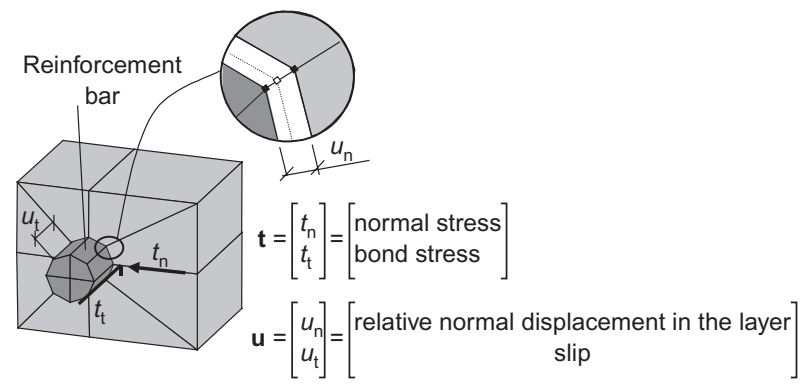

Fig. 1. Physical interpretation of the variables $t_{\mathrm{n}}, t_{\mathrm{t}}, u_{\mathrm{n}}$ and $u_{\mathrm{t}}$

used for deformed bars in Lundgren and Gylltoft ${ }^{1}$ or Lundgren. ${ }^{2}$ The main difference is that here the adhesion is taken into account. The reason is that for deformed bars, the adhesion contributes to only a small part of the bond, while for strands the ability to develop normal stresses during slip is much smaller. Thereby, the bond capacity owing to friction is strongly reduced, and thus the adhesion is no longer negligible.

In the model of the bond mechanism, elasto-plastic theory is used to describe the relations between the stresses and the deformations. The relation between the tractions $\mathbf{t}$ and the relative displacements $\mathbf{u}$ is in the elastic range

$$
\left[\begin{array}{c}
t_{\mathrm{n}} \\
t_{\mathrm{t}}
\end{array}\right]=\left[\begin{array}{cc}
D_{11} & \frac{\left|u_{\mathrm{t}}\right|}{u_{\mathrm{t}}} D_{12} \\
0 & D_{22}
\end{array}\right]\left[\begin{array}{l}
u_{\mathrm{n}} \\
u_{\mathrm{t}}
\end{array}\right]
$$

where $D_{12}$ normally is negative, meaning that slip in either direction will cause negative $t_{\mathrm{n}}$, that is compressive forces directed outwards in the concrete. The yield surface is defined by two functions, one describing the friction $F_{1}$, including the adhesion, $f_{\mathrm{a}}$

$$
F_{1}=\left|t_{\mathrm{t}}\right|+\mu\left(t_{\mathrm{n}}-f_{\mathrm{a}}\right)=0
$$

The other yield function, $F_{2}$, describes the upper limit, which is determined from the stress in the concrete resulting from the bond action. The maximum allowed tensile stress is set to $f_{\mathrm{a}}$, see Figure 2 .

$$
F_{2}=t_{\mathrm{t}}^{2}+\left(t_{\mathrm{n}}+c\right)\left(t_{\mathrm{n}}-f_{\mathrm{a}}\right)=0
$$

The yield surface is shown in Figure 2. For plastic loading along the yield function describing the upper limit, $F_{2}$, an associated flow rule is assumed. For the yield function describing the friction, $F_{1}$, a nonassociated flow rule is assumed, for which the plastic part of the deformations is

$$
\mathrm{d} \mathbf{u}^{p}=\mathrm{d} \lambda \frac{\partial G}{\partial \mathbf{t}}, G=\frac{\left|u_{\mathrm{t}}\right|}{u_{\mathrm{t}}} t_{\mathrm{t}}+\eta t_{\mathrm{n}}=0
$$

When the model was used for deformed bars, the dilatation $\eta$ was assumed to be constant, while for indented strands it was assumed to be a function of the slip (see the following subsection on 'input parameters'. For the

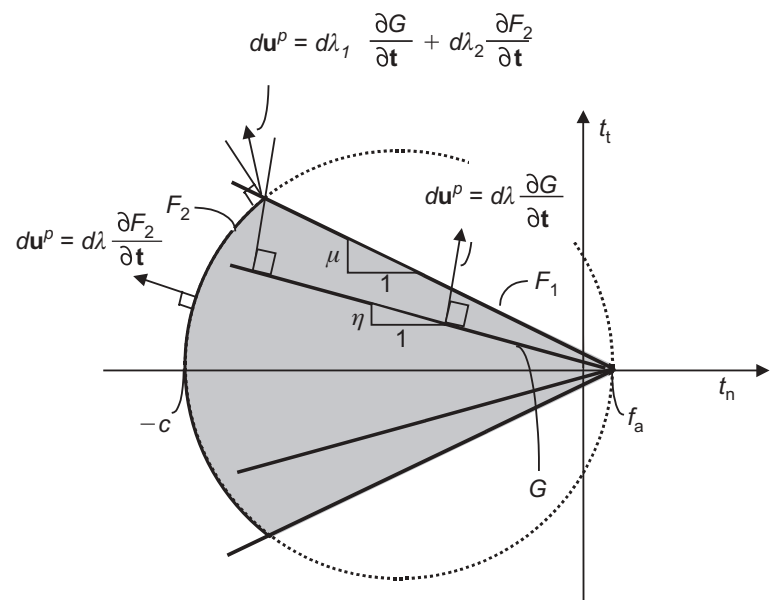

Fig. 2. The yield surface. The plastic part of the deformations, d $\mathbf{u}^{p}$, is given by an associated flow rule at the yield function describing the upper limit, $F_{2}$, and a non-associated flow rule at the yield function describing the friction, $F_{1}$

hardening rule of the model, a hardening parameter $\kappa$ is established. It is defined by

$$
\mathrm{d} \kappa=\sqrt{\mathrm{d} u_{\mathrm{n}}^{p_{2}}+\mathrm{d} u_{\mathrm{t}}^{p_{2}}}
$$

which means that the hardening parameter $\kappa$ is approximately equal to the applied slip. The variables $\mu, c$ and $f_{\mathrm{a}}$ in the yield functions are assumed to be functions of $\kappa$. The parameter $\eta$ is assumed to be a function of the slip. The original model was developed also for cyclic loading. However, this is not included in the present paper.

\section{Input parameters used for the indented strand}

As mentioned, the bond model was initially developed and calibrated for deformed bars. The pullthrough and push-in tests of indented strands in Gustavson $^{3-5}$ gave information about the bond mechanism in the strand-concrete interface, and were used to calibrate the parameters in the bond model. It was found that the initial bond response, that is the adhesion, makes a considerable contribution to the bond capacity of strands. Furthermore, it was found that the indentations of the strands affected the surrounding concrete in a somewhat different way compared with the ribs of deformed bars. Hence, there are two important changes in the input compared with the one chosen for deformed bars: $(a)$ since the adhesion is taken into account, a function $f_{\mathrm{a}}(\kappa)$ must be chosen; and $(b)$ the dilatation $\eta$ is assumed to be a function of the slip. While the input data for the elastic stiffness matrix D and the function $c(\kappa)$ were assumed to be the same for all of the strands studied, the functions $f_{\mathrm{a}}(\kappa), \mu(\kappa)$ and $\eta\left(u_{t}\right)$ were assumed to be different for the different strands tested and analysed.

The stiffnesses in the elastic stiffness matrix $\mathbf{D}$ were assumed to be related to the stiffness of the concrete. 
$D_{22}$ was recognised as the stiffness of the first part in a bond-slip curve. By comparing with results from experiments, it was chosen to be

$$
D_{22}=K_{22} E_{c}, K_{22}=3 \cdot 0 \mathrm{~m}^{-1}
$$

The stiffness $D_{12}$ was chosen to be

$$
D_{12}=K_{12} E_{c}, K_{12}=1 \cdot 8 \mathrm{~m}^{-1}
$$

The stiffness $D_{11}$ was assumed to be a function of the relative normal deformation, $u_{\mathrm{n}}$, as in recent work on deformed bars, see Lundgren. ${ }^{6}$ The function chosen is shown in Figure 3. The variable $c$ represents the stress in the inclined compressive struts as shown in Figure 3. The maximum of $c$ was therefore assumed to be the same as the uniaxial compressive strength of the concrete, as for deformed bars in Lundgren. ${ }^{6}$

The function $\mu(\kappa)$ describes how the relation between the bond stress and the normal splitting stress depends on the hardening parameter. It was assumed to decrease and eventually become constant at a low value with increasing slip in the interface. In Figure 4(a) the curve chosen for the strand with the original indenta-

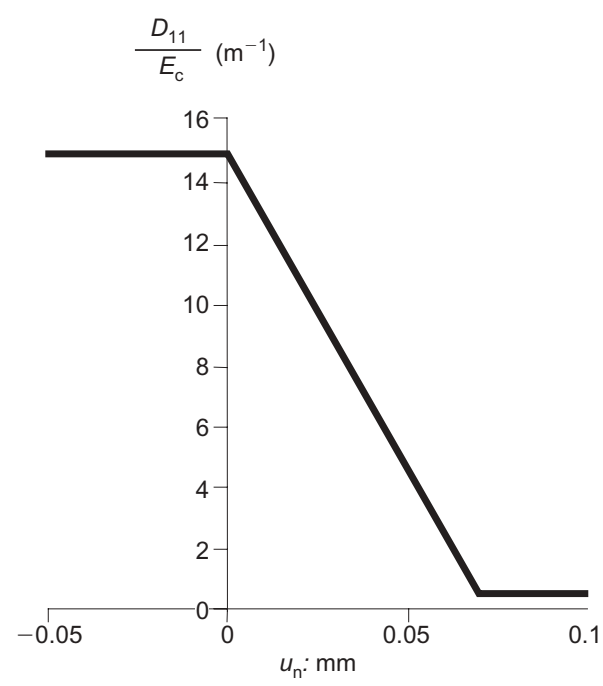

Fig. 3. The function chosen for the stiffness $D_{11}$

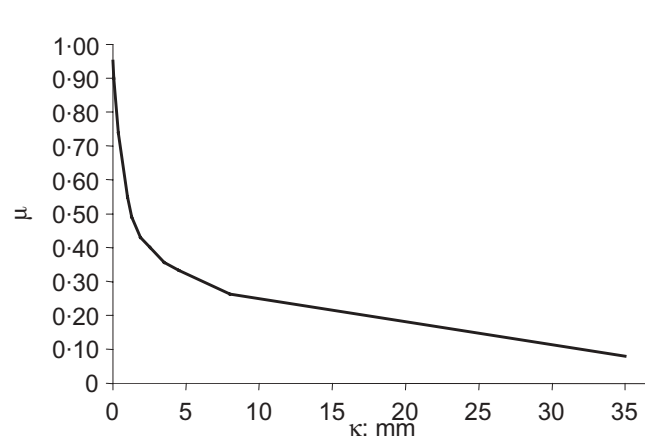

(a) tion is shown. The coefficient of friction depends mainly on the surface of the strand; however, changes in the concrete mix can have small effects.

The adhesive capacity of the strand-concrete interface can be limited either by the tensile capacity of the strand-concrete interface, or by the concrete itself. If the adhesion between the strand and the concrete is fully developed, failure will occur in the surrounding concrete at debonding. Otherwise, failure will occur at the interface. The adhesion, $f_{\mathrm{a}}(\kappa)$, is thus assumed to be lower than, or equal to, the tensile strength of the concrete, and to decrease down to zero for rather small slip, see Figure 4(b). How large the adhesion is depends both on the surface of the strand and on the concrete mix.

The choice of the parameter $\eta$ represents the largest difference between the calibrations for deformed bars and strands. During monotonic slip, the ribs of deformed bars will always push the surrounding concrete in the normal direction of the bar, until splitting or pull-out failure occurs. The indentations of the strand have a much lower height and extension than the ribs of the deformed bars. Therefore, the surrounding concrete is not always pushed in the normal direction of the strand during increasing slip. As the slip exceeds the length of the extruding concrete parts of the indented concrete channel, the strand does not need to make way in the concrete any more, since it fits in the indented concrete channel again, see Figure 5(a). This phenomenon is repeated in a wave pattern with increasing slip, as the strand indentation goes in and out of phase with the indented concrete channel. The influence of the indentation pattern is controlled by the $\eta$ parameter, which describes the dilatation of the strandconcrete interface, that is the capability of the interface to push the concrete away from the strand.

The $\eta$ parameter is positive when the strand and concrete surfaces tend to separate, and negative when they tend to move towards each other. When the strand and concrete surfaces tend to keep a constant distance from each other, the $\eta$ parameter is zero. The dilatation of the interface varies as the strand slips, and consequently

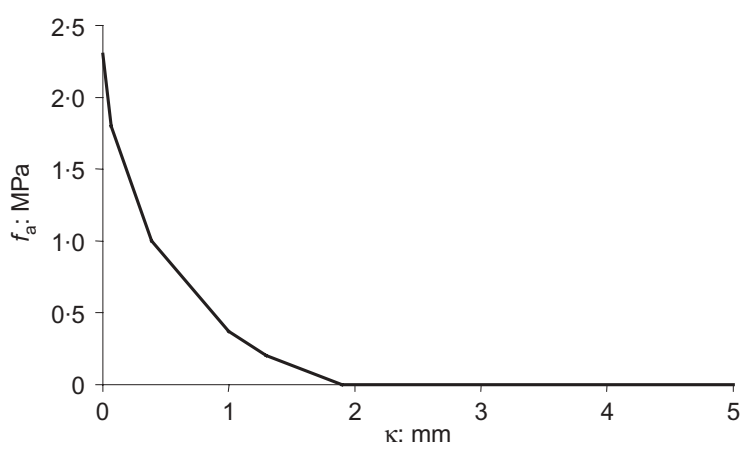

(b)

Fig. 4. (a) The coefficient of friction and (b) the tensile capacity of the interface, used in the FE analysis with the strand indented according to prEN $10138^{7}$ and a concrete with a compressive strength of $55 \mathrm{MPa}$

Magazine of Concrete Research, 2006, 58, No. 3 


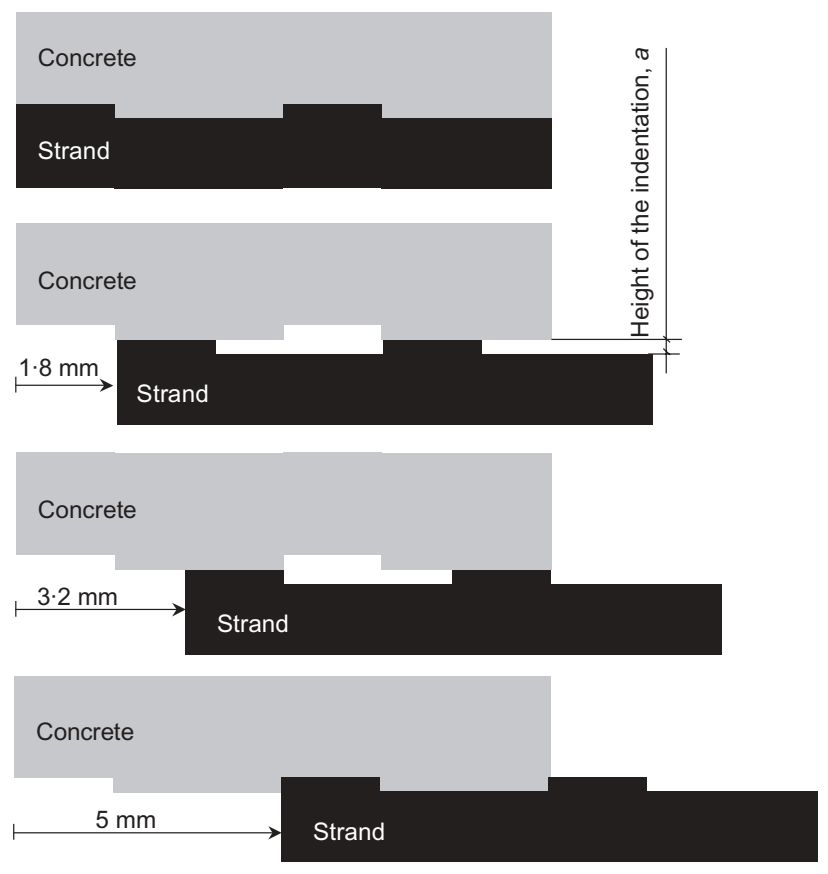

(a)

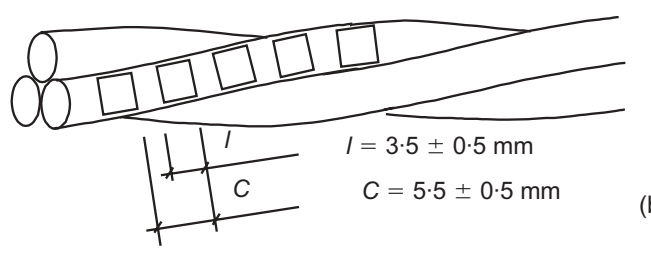

(b)

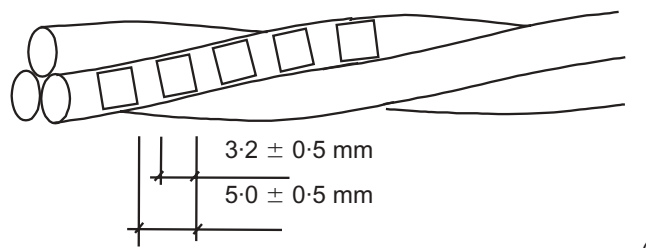

(c)

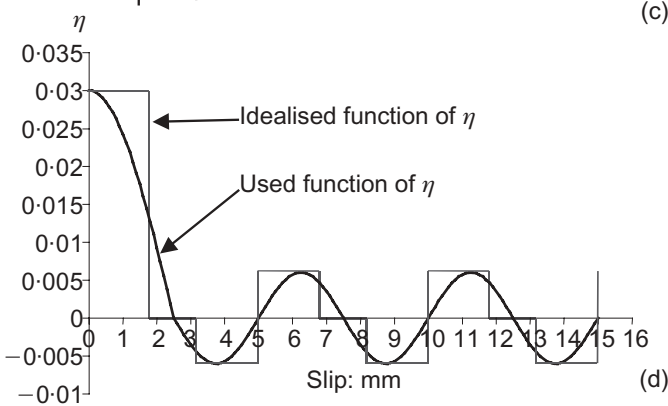

Fig. 5. (a) Relative normal displacement between the surrounding concrete and the strand owing to slip. Length of the strand indentation (b) in the direction of each wire and (c) in the direction of the entire strand. (d) The idealised and the used function $\eta\left(u_{\mathrm{t}}\right)$.

the $\eta$ parameter was assumed to be a function of the relative slip between the strand and the concrete. The length of the wire indentation in the direction of the wire (Figure 5(b)), given by prEN $10138,{ }^{7}$ was transformed to the length in the direction of the entire strand by use of measurement, see Figure 5(c). The idealised function of the $\eta$ parameter, given by the idealised theoretical dilatation of the interface, was changed to a function that was assumed to simulate more closely the real dilatation of the interface, see Figure 5(d). Thus, the dilatation $\eta$ is primarily dependent on the surface of the strand.

\section{FE analyses of tests with indented three-wire strand}

Pull-through and push-in tests were done by one of the authors, Gustavson, ${ }^{3,4,5}$ and the results were used for calibration of the bond model. The test results had some scatter of the obtained load and strain values, but the characteristics of the load-slip and tangential strain-slip relationships were equal within each tested configuration. A test result from each tested configuration, representative for all results, was chosen for the calibration. The test result used as reference is shown in all figures. If the scatter was large, the extremes at maximum and minimum are given. The test specimen consisted of a strand that was cast in a concrete cylinder surrounded by a steel tube. The steel tube had a diameter of $50 \mathrm{~mm}$, a height of $75 \mathrm{~mm}$ and a thickness of $1.0 \mathrm{~mm}$. The embedded length of the strand was $50 \mathrm{~mm}$. The tangential strains in the steel tubes were measured at one or three heights, together with the applied load and slip. Five or nine tests were done for each tested configuration used in the calibration of the bond model. In both the pull-through and the push-in tests, the strand and the concrete were able to rotate relative to each other during the tests by means of a thrust ball bearing. The test set-ups in the pull-through and the push-in tests are shown in Figure 6.

The pull-through and push-in tests were analysed with FE models. In all analyses, the concrete was modelled with a constitutive model based on non-linear fracture mechanics, using a rotating crack model based on total strain; see TNO. ${ }^{8}$ For the tension softening, the curve by Hordijk et al. was chosen, as described in TNO. ${ }^{8}$ The hardening in compression was described by the expression of Thorenfeldt et al. ${ }^{9}$ For the compressive cylinder strength, Young's modulus, and the fracture energy of the concrete, the measured values were used, see Gustavson. ${ }^{3,4,5}$ Other necessary material data for the concrete, such as the tensile strength and Poisson's ratio, were estimated according to the expressions in Comité Européen de Béton (CEB) ${ }^{10}$ from the compressive cylinder strength. The compressive cylinder strength of the concrete in the specimens used for the pull-through and push-in tests was 53-55 and 21-26 MPa respectively. The constitutive behaviour of the reinforcement steel was modelled by the von Mises 


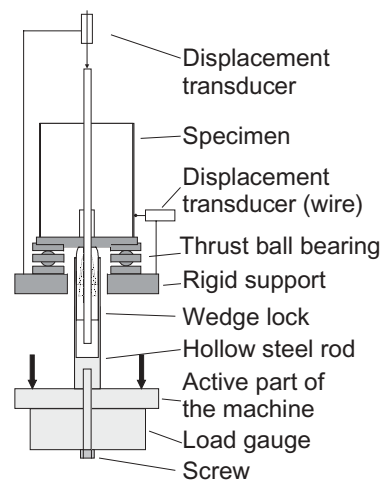

(a)

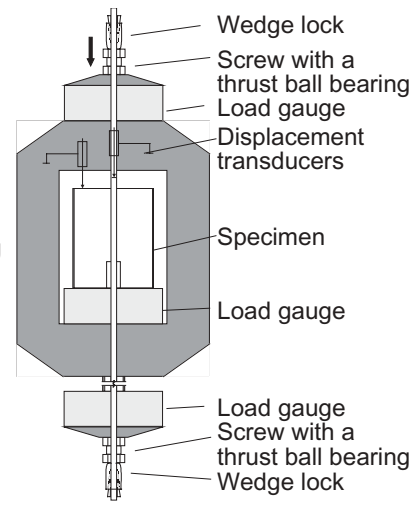

(b)
Fig. 6. The set-up in (a) the pull-through tests and (b) the push-in tests

yield criterion with associated flow and isotropic hardening. The elastic modulus of the reinforcement was assumed to be $200 \mathrm{GPa}$ according to the manufacturer Fundia Hjulsbro AB.

All of the FE models were axisymmetric with the assumption of four radial cracks. The physical rotation of the strand was not taken into account in the FE model. The three-wire strand used in the tests was modelled as one uniform strand with equal nominal diameter. The reason for this simplification of the element mesh, that is not modelling the winding of the strand, is that the mesh would be too complicated when modelling entire concrete members. However, the effect of the bond response owing to the relative rotation between the strand and the concrete is taken into account in the behaviour of the bond model.

\section{Pull-through tests}

The input parameters given in the preceding section 'Input parameters used for the indented strand' were used in the FE analysis of the pull-through tests. The load-slip relation from the finite element analyses was compared with the test results, see Figure 7. The bond response was well simulated until the first local mini- mum in the load-slip relationship, at a slip of approximately $5 \mathrm{~mm}$. The bond response in the FE analysis was slightly underestimated for increasing slip, but the characteristic wave pattern of the load-slip relationship was well captured. The change in the bond bearing from pure adhesion to a combination of adhesion, friction and mechanical action at a slip of $0.05 \mathrm{~mm}$ is clear in Figure 7(b). The maximum bond capacity is reached during a plateau in the load-slip relation. The plateau begins when the slip is equal to the length of the extruding parts of the strand indentation $(1.8 \mathrm{~mm})$, and ends at a slip of approximately $2.5 \mathrm{~mm}$. The local minima in the load-slip relation occur at slip values at which the indented strand and concrete channel fit, that is, are in phase - meaning that the slip value corresponds to a value that is a multiple of $5 \mathrm{~mm}$.

The measured tangential strains in the encasing steel tube were compared with the corresponding strains in the FE analysis, see Figure 8. The strain development from no slip to a slip of $5 \mathrm{~mm}$ is well captured in the FE analysis. The following development of the strain in the FE analysis differs slightly from the measured strain.

\section{Push-in tests}

The effect of the longitudinal steel stress on the local bond behaviour was examined by the results from the push-in tests and FE analysis. The push-in tests were done at a compressive concrete strength of 21-26 MPa and consequently the input of the functions $\mu(\kappa)$ and $f_{\mathrm{a}}(\kappa)$ had to be changed and calibrated. The variation of the interface tensile capacity and the friction used as input parameters, given by the calibration, is shown in Figure 9. The other input parameters were chosen to be the same as given from the calibration of the pullthrough test.

The push-in test had a maximum slip of approximately $6 \mathrm{~mm}$. The load-slip relation and the tangential strain-slip relation from the test and the FE analysis are compared in Figure 10. The load-slip relation is well captured in the FE analysis until the maximum bond capacity is reached. The tangential strain in the

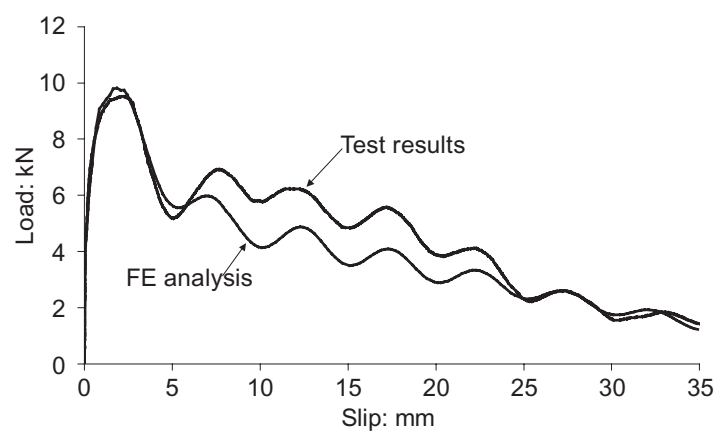

(a)

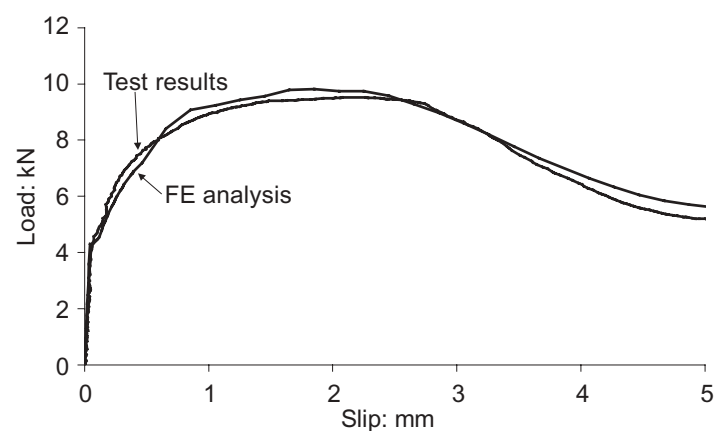

(b)

Fig. 7. Comparison of the load-slip relation of an indented three-wire strand, indentation according to prEN $10138,{ }^{7}$ in a pull-through test and the FE analysis. Magnified slip scale in (b) 


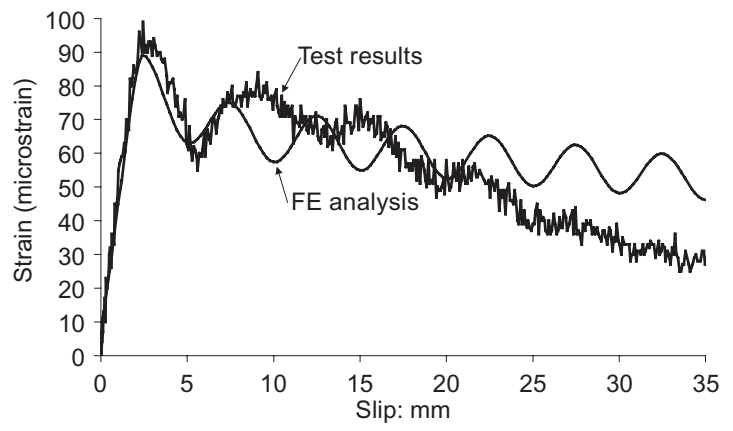

(a)

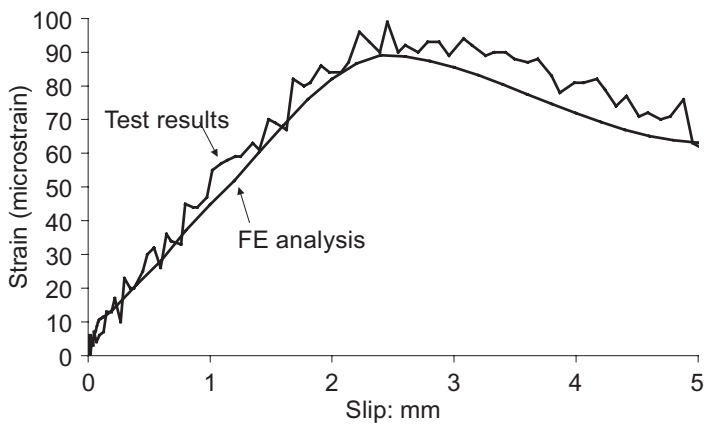

(b)

Fig. 8. Comparison of the tangential strain-slip relation of an indented three-wire strand, indentation according to prEN $10138,{ }^{7}$ in pull-through tests and the FE analysis. Magnified slip scale in (b)

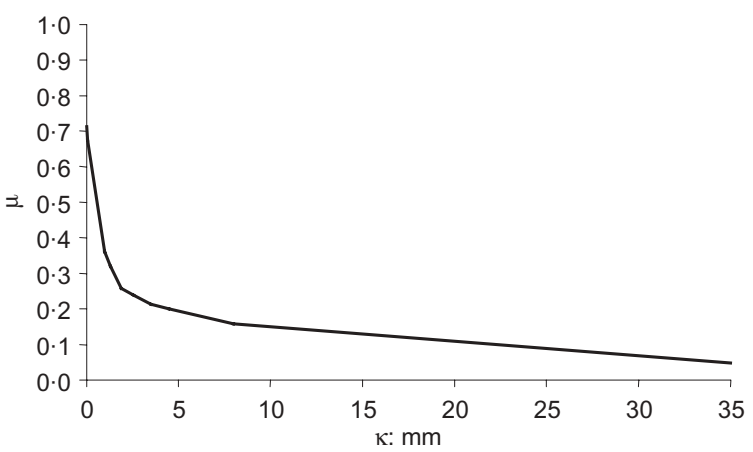

(a)

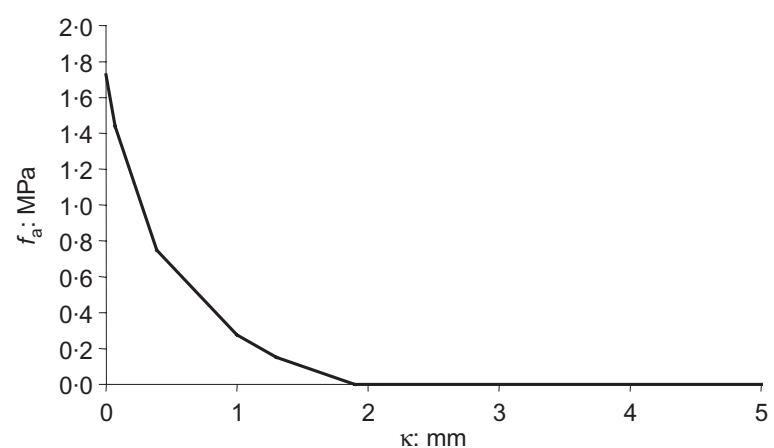

(b)

Fig. 9. (a) The coefficient of friction and (b) the tensile capacity of the interface, used in the FE analysis with the strand indented according to prEN $10138^{7}$ and a concrete with a compressive strength of $26 \mathrm{MPa}$

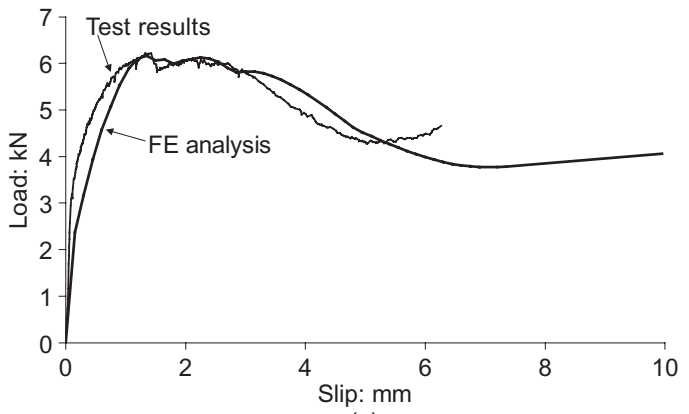

(a)

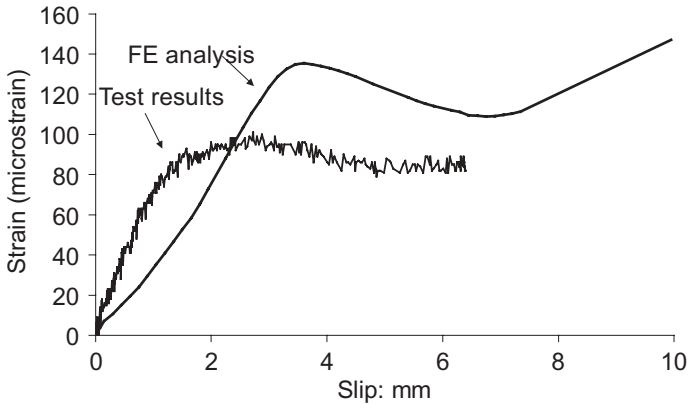

(b)

Fig. 10. Comparison of (a) the load-slip relation and (b) the tangential strain-slip relation of an indented three-wire strand, indentation according to prEN $10138,{ }^{7}$ in a push-in test and a FE analysis

FE analysis is larger than the strain measured in the tests. There are two possible explanations for this: it is caused either by the shrinkage of the concrete in the test or by the difference of the Poisson effect between a uniform bar, as in the analysis, and a three-wire strand as in the tests.

\section{FE analyses of tests with variations of the strand properties}

In the pull-through tests reported in Gustavson ${ }^{3,4,5}$ tests with variations of the strand or the concrete properties were done in order to study the influence of different changes in the bond behaviour. A comparison between the results from the tests and FE analyses with the bond model was made in order to see if the model was able to capture the different bond behaviours, by changing the input parameters used in a natural and reasonable way.

\section{Surface roughness}

The influence of the surface roughness on the bond behaviour was examined by comparing the results from pull-through tests of a smooth three-wire strand with a smooth three-wire strand that had a sandblasted surface. Results from an untreated indented three-wire strand and a teflon-coated three-wire strand were also

Magazine of Concrete Research, 2006, 58, No. 3 
compared. The teflon coating was used, as far as possible, to decrease the roughness of the strand surface, while sandblasting was used to maximise the roughness of the strand surface. A smooth strand was used in the case of a maximised strand surface roughness, by sandblasting, in order to obtain clear results. If an indented strand had been used, the sandblasting would have damaged the indentation to an uncontrollable extent. This would have given results that could be hard to interpret. The tests were analysed with the bond model, and the input parameters were calibrated until proper behaviour of the strand-concrete interface was achieved.

The input parameters used for the two cases of a sandblasted and an untreated smooth strand are shown in Figure 11. The $\eta$ parameter was set equal for both the smooth strands (a bi-linear function with $\eta$ equal to 0.015 at no slip and 0 at a slip of $2.5 \mathrm{~mm}$ ). The friction function in the case of a smooth wire with no surface treatment was given a lower value. The function of the interface tensile capacity was also changed between the two cases. It was assumed that the tensile capacity of the concrete was limiting the adhesive capacity in the case of a sandblasted wire surface. The tensile capacity of the wire surface with no treatment was then calibrated by use of the test results. A comparison of the load-slip and tangential strain-slip relationships in the tests and the analyses with the sandblasted and the untreated smooth strand is given in Figures 12 and 13 respectively.

The input parameters used in the analyses of the tests with an untreated indented strand and a teflon-coated strand are given in Figure 14. The function of the $\eta$ parameter was set equal in both cases since the indentations of the strands were equal. The changes of the input parameters were done for the function of the friction, $\mu$, and the tensile capacity of the interface. Both parameters were given a lower value in the case of teflon coating of the wire surface.

A comparison of the load-slip relation in the tests and the FE analyses with the teflon-coated and the untreated indented three-wire strand is given in Figure 15. The tangential strain-slip relation in the tests and the FE analyses is compared in Figure 16.

A change of the roughness of the wire surface will affect both the adhesion and the subsequent friction in the strand-concrete interface. The reduced friction in the case of the teflon-coated indented strand seems to affect the wave pattern of the load-slip relation. This is not captured in the FE analysis. However, it can be concluded that the difference in the bond behaviour owing to a change of the strand surface can be modelled and understood by considering the adhesion and the friction parameter.

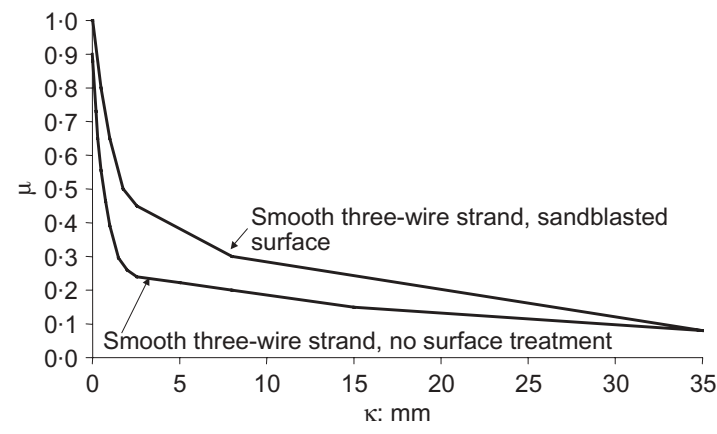

(a)

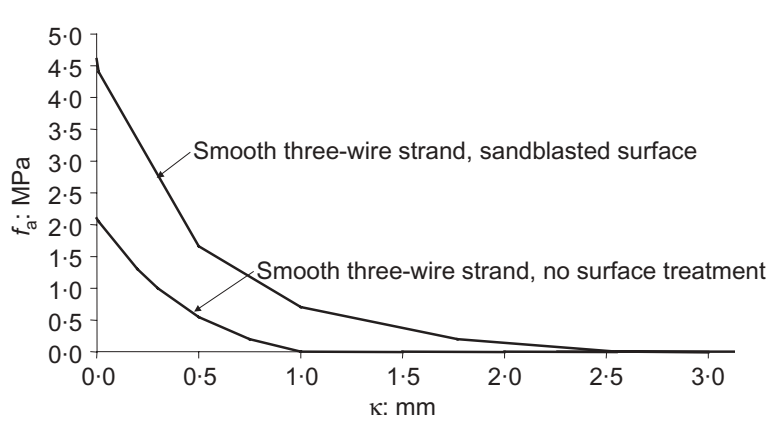

(b)

Fig. 11. (a) The functions of the $\mu$ parameter and (b) the function for the tensile capacity of the interface

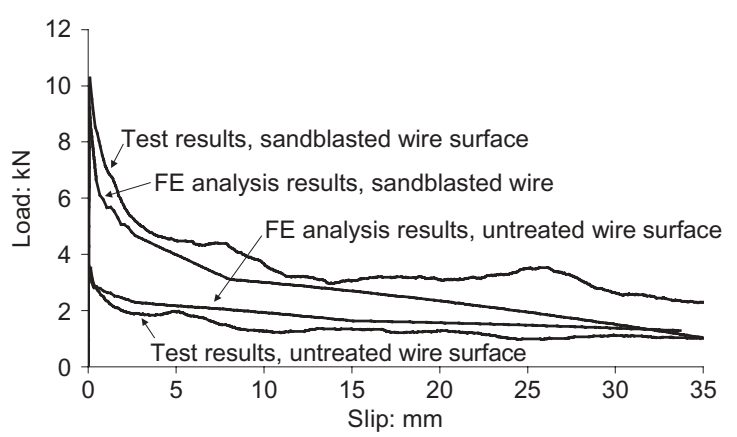

(a)

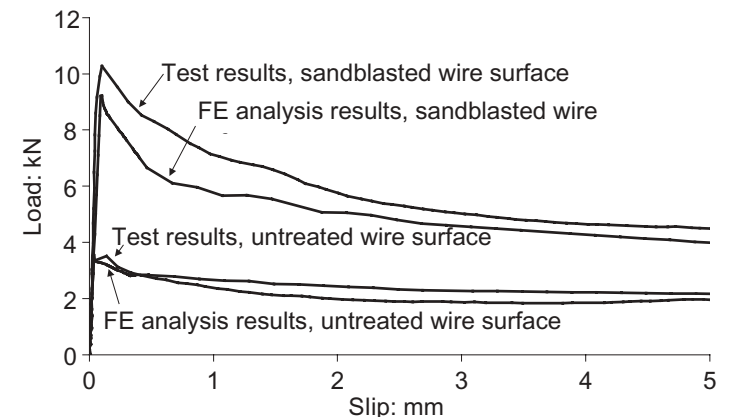

(b)

Fig. 12. Load-slip relation from tests and FE analysis of a smooth three-wire strand with sandblasted and untreated wire surface. Magnified slip scale in (b). 


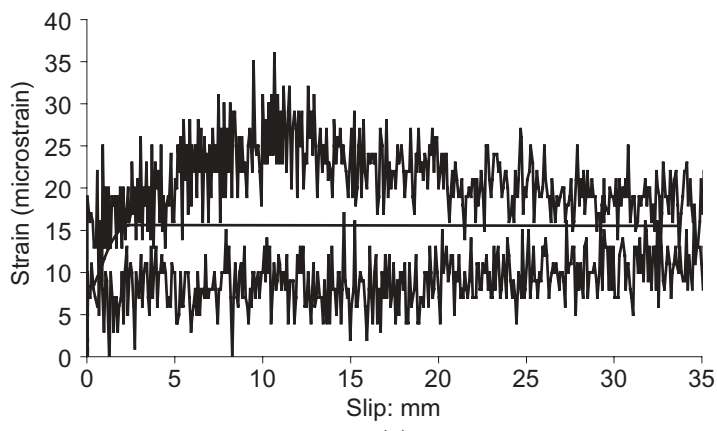

(a)

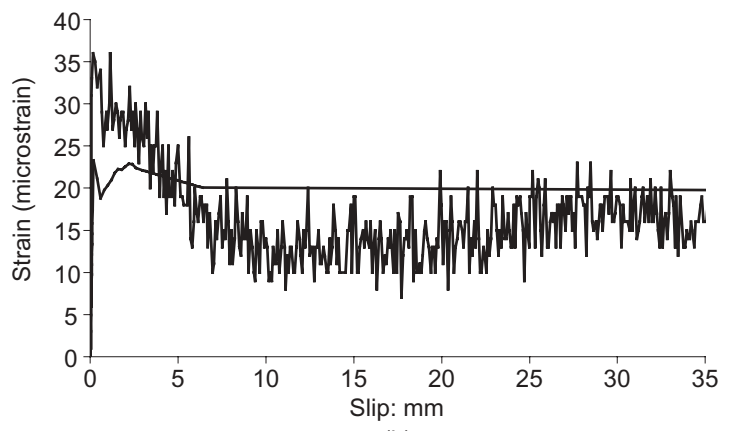

(b)

Fig. 13. Tangential strain-slip relation in the tests and the FE analysis for a smooth three-wire strand with (a) untreated wire surface and (b) sandblasted wire surface.

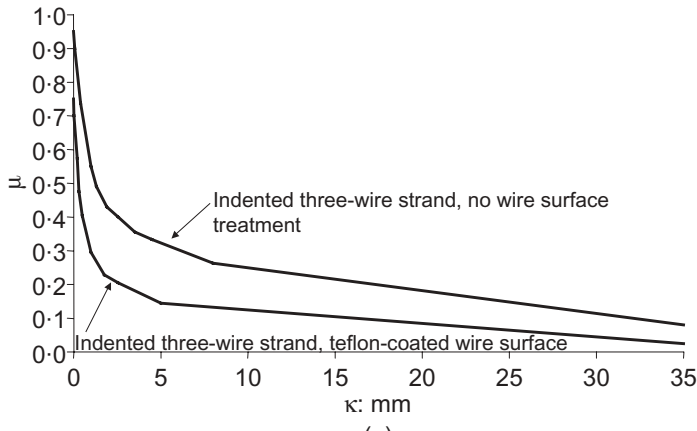

(a)

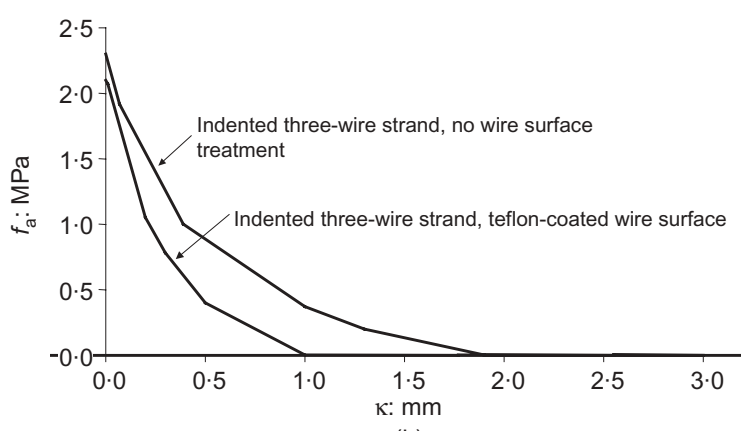

(b)

Fig.14. (a) The functions of the $\mu$ parameter and (b) the function for the tensile capacity of the interface

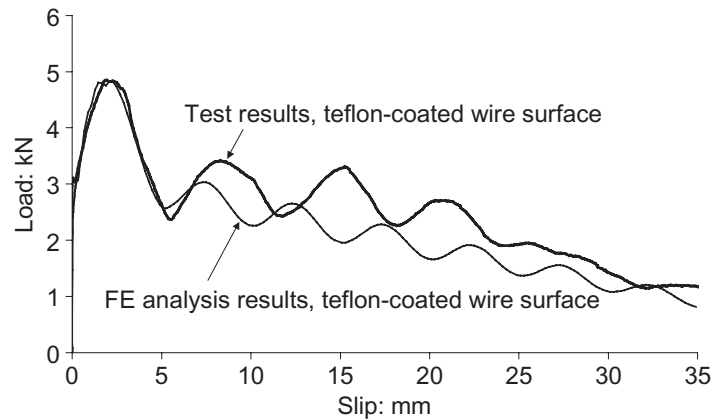

(a)

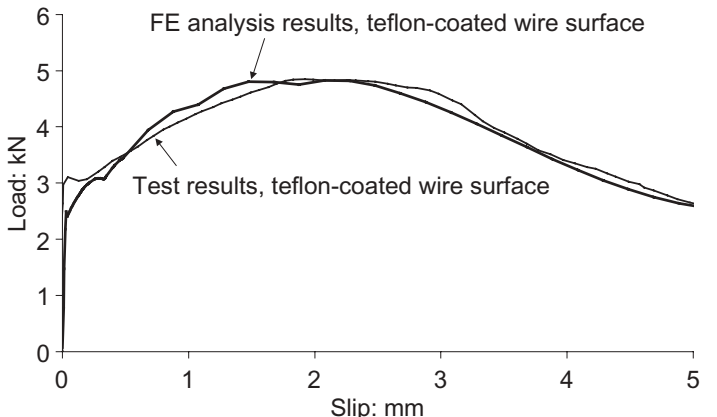

(b)

Fig. 15. Load-slip relation from tests and FE analysis of an indented three-wire strand with teflon-coated wire surface. Magnified slip scale in (b)

\section{Indentation}

The variation in the bond behaviour attributed to a variation of the strand indentation is shown by test results from a strand with an indentation according to prEn $10380^{7}$ and a strand with a modified indentation. The strands had different longitudinal placement of the indentation (see Figure 17), while the heights of the indentations were equal $(0.06 \mathrm{~mm})$.

The function for the tensile capacity of the interface was set equal in both analyses. The friction was a bit higher in the case of modified indentation. The physical explanation was assumed to be that the concrete indentations wore down at a slower rate when the modified indentation was used, as the length of the concrete indentations was increased, see Figure 18(a). The main difference between the two analyses was the input of the $\eta$ function. The $\eta$ function was changed according to the geometry of the pattern of the modified indentations, see Figure 18(b). The load-slip and tangential strain-slip relations of the strand with the modified indentation in the tests and the FE analysis were compared, see Figures 19 and 20.

It can be concluded that the indentation of the strand strongly affects the bond behaviour, and that the $\eta$ 


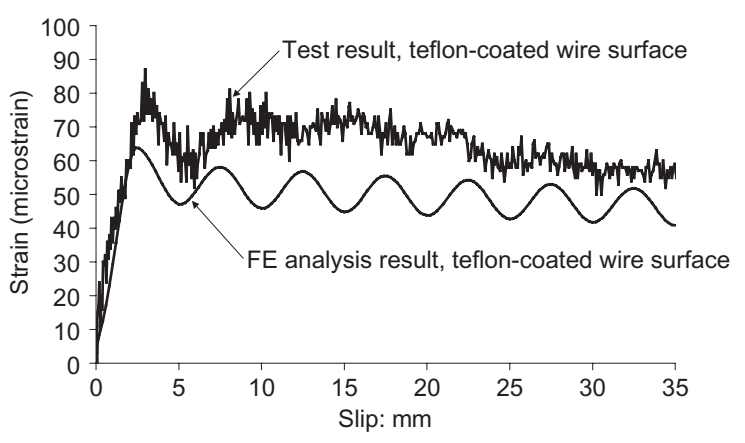

Fig. 16. Tangential strain-slip relation in the tests and the FE analysis for an indented three-wire strand with tefloncoated wire surface

parameter can be used to simulate the influence of the indentation geometry. The wave pattern in the loadslip and tangential strain-slip relations is captured both in the case of an indentation according to prEN 10138 and in the case of a modified indentation. This is attributed to the fact that the indentation pattern of the strand surface and the slip are connected by the function of the $\eta$ parameter.

\section{Modelling used to understand the bond mechanisms}

An FE analysis of both the pull-through and the push-in test set-ups was done with equal input parameters in order to examine further the facilities of the bond model. The load-slip relation and the tangential strain-slip relation were compared, see Figure 21. The difference in the two test set-ups is that a change of the local longitudinal steel stress is present in the push-in test. This implies that there is an increase of the normal compressive stress in the strand-concrete interface, and
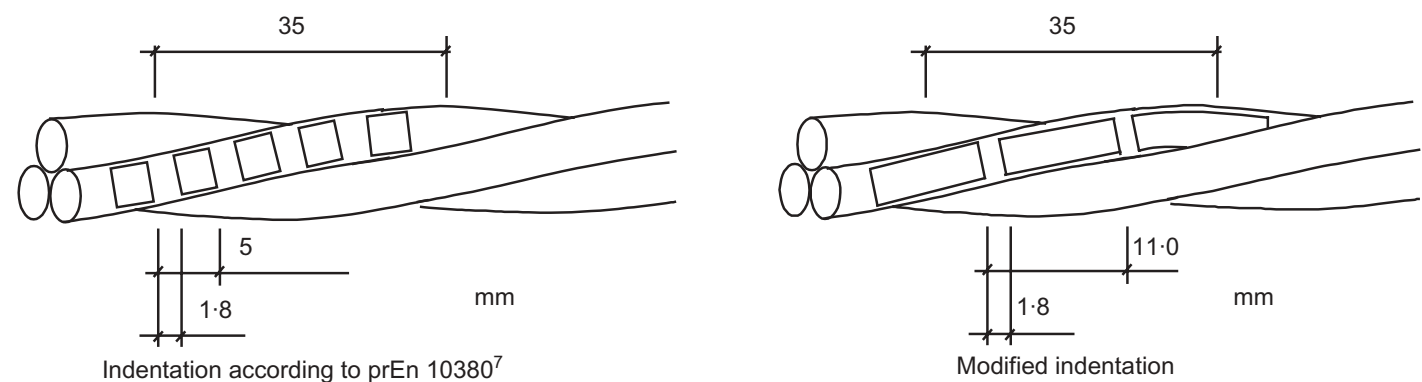

Fig. 17. The two different strand indentations used in the tests and analyses

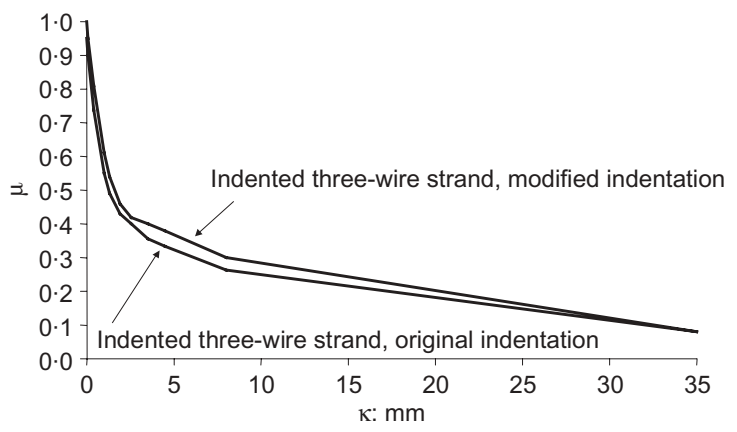

(a)

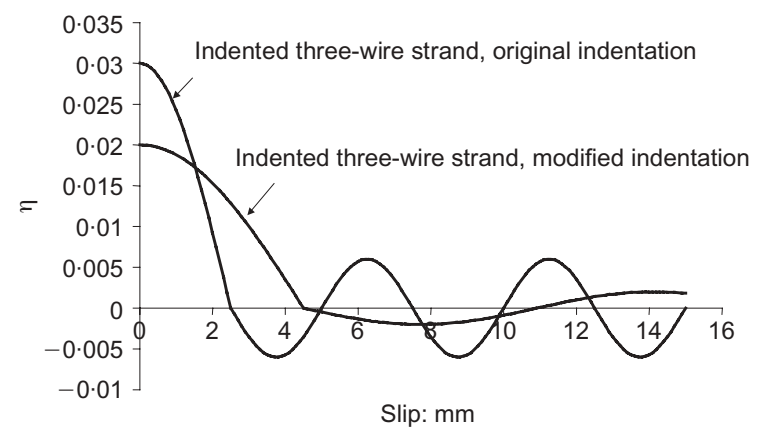

(b)

Fig. 18. (a) The functions of the $\mu$ parameter and (b) the function of the $\eta$ parameter

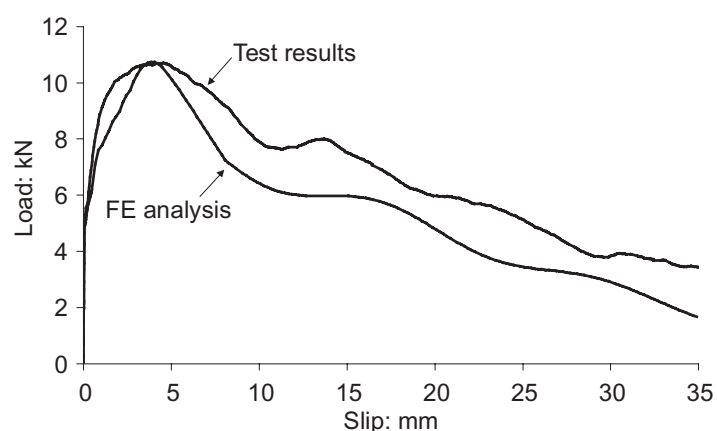

(a)

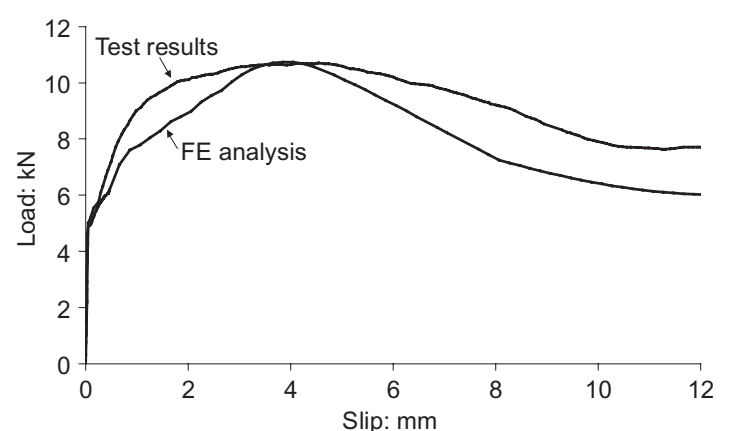

(b)

Fig. 19. Load-slip relation from tests and FE analysis of a three-wire strand with modified indentation. Magnified slip scale in (b) 


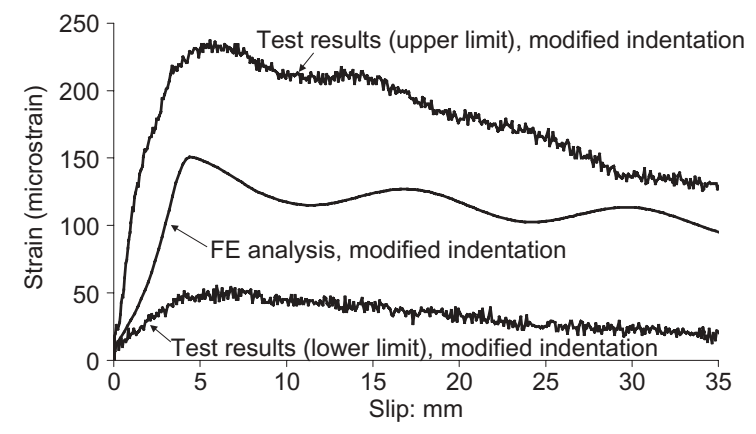

Fig. 20. Tangential strain-slip relation in the tests and the FE analysis for a three-wire strand with modified indentation

this should influence the bond response, as the bond model considers the stress state in the interface. It is clear from Figure 21(a) that the change of local steel stress affects the adhesive capacity as well as the shape of the load-slip relation at the maximum bond capacity.

The length of the peak plateau in the bond response is increased in the analysis of the push-in test. The reason for this emerges when the tangential strain-slip relation in Figure 21(b) is examined. The increase of the strand diameter during slip creates an increasing compressive normal stress that is added to the normal stress variation owing to the strand indentation, that is, the function of the $\eta$ parameter. A tendency to an increased length of the peak plateau in the load-slip relation could be found when the load-slip relations from the pull-through and push-in tests were compared, see Figure 22(a). The difference in bond capacity owing to adhesion could not be compared in the test results, as the concrete had different compressive strength at the time of testing. However, the increased adhesive capacity in the analyses could be explained by examination of the development of elastic stresses in the stress space of the interface. The elastic stress state of the interface will be prolonged, since the increased normal stress changes the 'path' of the stress develop- ment and this results in an increased bond stress when the yield surface is reached, see Figure 22(b). Hence, the normal stress owing to the increasing strand diameter will increase the slip value where the peak of the strain in the tangential strain-slip relation occurs, see Figure 21(b).

The path of the stress development within the stress space of the interface reveals a lot of information concerning the influencing parameters and the involved bond mechanisms. The paths of the stress development during a pull-through test for an indented three-wire strand (indentation according to prEN 10138 ${ }^{7}$ ) and a ribbed bar are compared in Figure 23. Splitting failure is avoided in both analyses in the comparison. The stress path of the ribbed bar shows that the stress development at pull-out failure is limited by the yield surface, $F_{2}$, which is built up by the tensile and compressive strength of the concrete. However, for an indented strand the yield surface, $F_{2}$, is never reached. Hence, the concrete strength is a strongly influencing parameter for the pull-out failure of ribbed bars but not for indented strands. The maximum bond stress of strands is not set by the yield surface $F_{2}$. The maximum bond stress is set by the parameter controlling the development of normal stress, $t_{\mathrm{n}}$, that is the $\eta$ parameter. Hence, the maximum bond capacity is strongly influenced by the indentations of the strand.

\section{Conclusions}

Knowledge of the bond mechanisms present between three-wire strands and concrete, and some of the affecting parameters, was gained by a parallel use of results from tests and numerical modelling. The three mechanisms of adhesion, friction and mechanical action were found to determine the bond behaviour of the strand. The initial bond response was mainly attributed to adhesion in the strand-concrete interface, which caused a bond capacity of almost half of the maximum bond capacity. The slip at the maximum bond capacity,

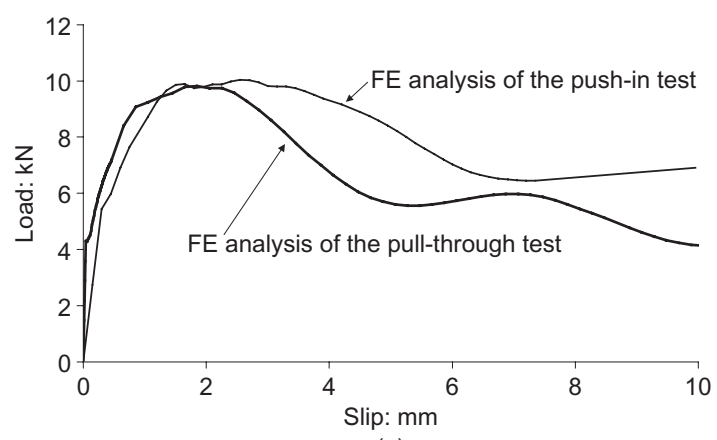

(a)

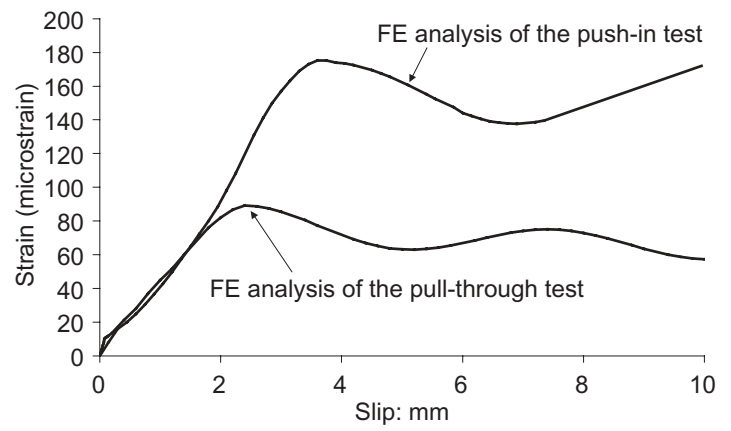

(b)

Fig. 21. Comparison of (a) the load-slip relation and (b) the tangential strain-slip relation of an indented three-wire strand, indentation according to prEN 10138, ${ }^{7}$ in the FE analyses of the pull-through and push-in tests 


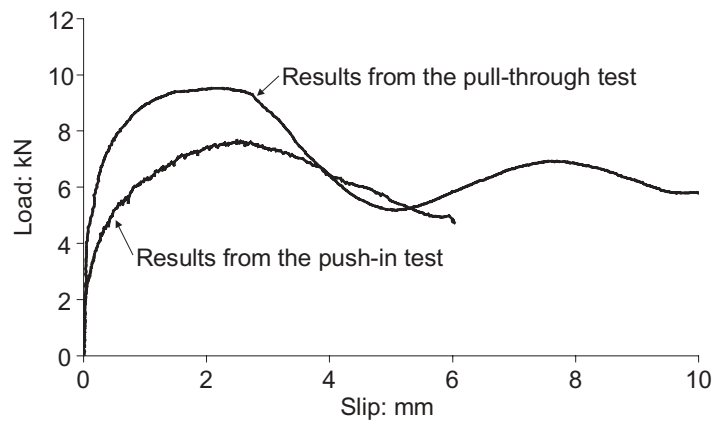

(a)

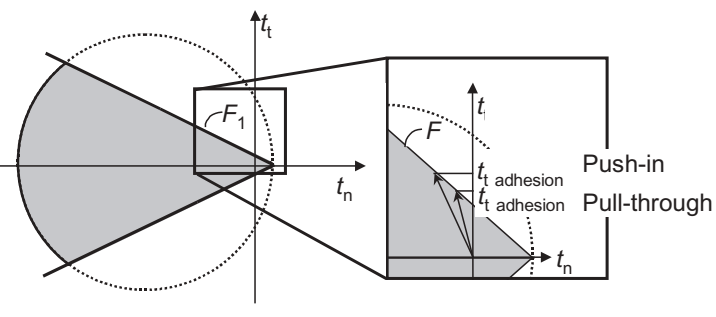

(b)

Fig. 22. (a) The load-slip relation in the pull-through and push-in tests. (b) Influence on the adhesive bond capacity owing to increased normal compressive stress in the strand-concrete interface

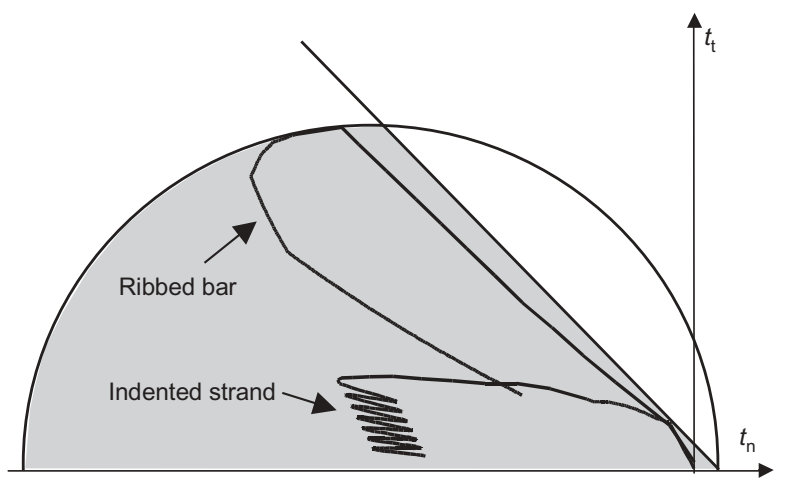

Fig. 23. Paths of the stress development for an indented strand and a ribbed bar in the stress space of the reinforcement concrete interface. Splitting failure is avoided

and also the maximum bond capacity, were determined by the indentation pattern.

It was concluded that, as long as splitting failure is avoided, the maximum bond capacities of ribbed bars and indented strands are limited by two completely different mechanisms. For ribbed bars the maximum bond capacity is limited by a shear failure of the concrete between the ribs, which corresponds to the same capacity as compressive failure of the inclined struts surrounding the bar. The maximum bond capacity of indented strands is limited by the adhesion in the strand-concrete interface, or by the ability of the strand to create compressive normal stresses in the strandconcrete interface. Hence, while the maximum bond capacity of ribbed bars depends on the compressive strength of the concrete, the maximum bond capacity of strands depends on the properties of the strand surface and indentation.

\section{References}

1. Lundgren K. and Gylltoft K. A model for the bond between concrete and reinforcement. Magazine of Concrete Research, 2000, 52, No. 1, 53-63.

2. Lundgren K. Modelling of Bond: Theoretical Model and Analyses. Chalmers University of Technology, Division of Concrete Structures, Göteborg 1999, Report 99:5.

3. Gustavson R. Steel-Encased Pull-Through and Push-In Tests of Indented Three-Wire Strands. Chalmers University of Technology, Department of Structural Engineering, Göteborg 2001, Report 01:2, p.182.

4. Gustavson R. Pull-Through Tests of Strands with Various Surface Treatments and Geometries. Chalmers University of Technology, Department of Structural Engineering, Göteborg 2001, Report 01:6, p.182.

5. Gustavson R. Steel-Encased Pull-Through Tests of Three-Wire Strands With Different Properties of the Strand and the Concrete. Report 02:1, Concrete Structures, Chalmers University of Technology, Göteborg 2002, 205 pp.

6. Lundgren K. Modelling the effect of corrosion on bond in reinforced concrete. Magazine of Concrete Research, 2002, 54, No. 3, 165-173.

7. Comité Européen de Normalisation. Design of prestressing steel - Part 3: Strand, prEN 10138. CEN, Brussels.

8. TNO Building and Construction Research. DianA Finite Element Analysis, User's Manual release 7. TNO Building and Construction Research, Hague 1998.

9. Thorenfeldt E., Tomaszewicz A. and Jensen J. J. Mechanical properties of high-strength concrete and application in design. Proceedings of Symposium on Utilization of High Strength Concrete, Stavanger, Norway. Tapir N-7034, Trondheim, 1987.

10. Comité Européen de Béton. CEB-FIP Model Code 1990. Bulletin d'Information, No. 213/214, CEB, Lausanne 1993.

Discussion contributions on this paper should reach the editor by 1 October 2006 